\title{
Avaliação da coluna vertebral: relação entre gibosidade e curvas sagitais por método não-invasivo
}

\author{
Spine evaluation: Determination of the relationship between thoracic \\ spinal deformity and sagittal curves by a noninvasive method
}

\author{
Dalva Minonroze Albuquerque Ferreira \\ Cíntia Girardi Fernandes \\ Marcela Regina Camargo \\ Célia Aparecida Stelluti Pachioni ${ }^{1}$ \\ Cristina Elena Prado Teles Fregonesi \\ Cláudia Regina Sgobbi Faria ${ }^{1}$
}

\section{Universidade Estadual Paulista. Faculdade de Ciencias e Tecnolo- gia. Departamento de Fisioterapia. Presidente Prudente, SP. Brasil}

2 Universidade Estadual Paulista. Faculdade de Ciências e Tecnologia. Bacharel em Fisioterapia. Campus de Presidente Prudente, SP, Brasil.

3 Universidade Estadual Paulista. Faculdade de Ciências e Tecnologia. Mestre em Fisioterapia. Campus de Presidente Prudente, SP, Brasil.

Recebido em 06/10/09 Revisado em 23/11/09 Aprovado em 18/03/10
Resumo - Os objetivos do estudo foram avaliar o alinhamento, no plano sagital, da coluna de indivíduos com alterações na medida da gibosidade, comparando com um grupo sem alterações; testar a confiabilidade do instrumento utilizado e verificar se existem correlações entre as medidas da gibosidade e os valores das curvaturas vertebrais. Foram avaliados 40 jovens, divididos em grupo controle - ausência ou presença de gibosidades inferiores a 0,5 $\mathrm{cm}$ na curvatura torácica e $0,7 \mathrm{~cm}$ na lombar $(\mathrm{n}=20)$ e, grupo experimental - gibosidades superiores às descritas $(n=20)$. A gibosidade e as curvaturas no plano sagital foram mensuradas com um instrumento adaptado a um nível d'água e o teste de Adams. As coletas foram realizadas em duas datas distintas, nos dois grupos. Após aplicação do teste MannWhitney não foi encontrada diferença entre as ocasiões de coletas e, emparelhando-se os grupos, foi encontrada diferença apenas na medida cervical. Na verificação de existência de relação entre as medidas coletadas, foi encontrada correlação linear (Spearmann) no grupo controle - curvatura torácica e gibosidade torácica; em ambos os grupos - curvaturas torácica e lombar; e no grupo experimental - gibosidade torácica e as curvaturas lombar e sacral e, curvatura sacral e curvaturas torácica e lombar. Pôde-se concluir que a medida da gibosidade tem relações com as curvaturas no plano sagital. Por ser um método confiável, simples e acessível, pode ser reproduzido sem altos custos financeiros e sem causar prejuízo à saúde do paciente.

Palavras-chave: Escoliose; Coluna vertebral; Curvaturas da coluna vertebral; Cifose; Lordose.

Abstract - The objectives of this study were to compare sagittal plane alignment between subjects with spinal deformities and a group presenting no changes; to test the reliability of the tool used, and to determine the existence of correlations between spinal deformity and sagittal curvature measures. Forty young subjects were divided into two groups: a control group $(n=20)$ presenting no changes or spinal deformity less than $0.5 \mathrm{~cm}$ in the dorsal curvature and $0.7 \mathrm{~cm}$ in the lumbar curvature, and an experimental group $(n=20)$ with spinal deformities greater than those described for the control group. Spinal deformity and sagittal plane curvatures were measured using a water level-based tool and by the Adams test. Data were collected from the two groups on two distinct occasions. The Mann-Whitney test showed no difference between sampling times. A significant difference between the two groups was only observed in terms of cervical curvature. Spearman's test revealed a linear correlation between dorsal curvature and dorsal spinal deformity in the control group, between dorsal and lumbar curves in the two groups, and between dorsal spinal deformity and lumbar and sacral curves and between sacral curvature and dorsal and lumbar curves in the experimental group. In conclusion, spinal deformity measurement is associated with sagittal plane curvatures. The method proposed here is reliable, simple and accessible and can be reproduced without high costs and damage to the patient's health.

Key words: Scoliosis; Spine; Spinal curvature; Kyphosis; Lordosis. 


\section{INTRODUÇÃO}

A coluna vertebral, quando vista de perfil, exibe quatro curvaturas: uma cervical (superior - do occipital ao áxis e inferior - do platô do áxis ao platô superior da primeira vértebra torácica), uma torácica, uma lombar e uma sacral. Essas curvaturas, visíveis apenas no plano sagital, são fisiológicas e, aliadas à existência dos discos e ligamentos vertebrais, permitem à coluna a absorção das forças compressivas verticais ${ }^{1}$. Em vista frontal, a coluna deve se apresentar retilínea, sem desvios laterais, com apófises e corpos vertebrais alinhados. Curvas perceptíveis nesse plano caracterizam a escoliose, contudo, essa deformidade ocorre com desvios nos três planos de referência ${ }^{2-4}$. Os desvios laterais das vértebras são acompanhados por rotações axiais vertebrais no plano transverso que, embora não sejam bem entendidas, são determinantes para o início e progressão da escoliose ${ }^{3,5}$.

Nas escolioses estruturais, esse componente rotacional é caracterizado pela presença de uma proeminência (gibosidade) no lado convexo da cur$\mathrm{va}^{6}, \mathrm{com}$ os corpos vertebrais rodados no sentido da convexidade. A gibosidade torácica aparece devido à rotação da caixa torácica (subjacente à rotação vertebral) e a gibosidade lombar ou a tóraco-lombar ocorre por aumento do volume e proeminência da musculatura. Em ambos os casos, a gibosidade pode ser correlacionada com a magnitude da deformidade espinhal ${ }^{7,8}$. As curvas escolióticas surgem, na maioria das vezes, durante a fase de aceleração do crescimento vertebral, o que torna crianças e adolescentes mais susceptíveis a desenvolvê-las. A progressão da deformidade ocorre devido à desordem neurogênica da musculatura paraespinal; desequilíbrio muscular entre os dois lados da coluna; desordens proprioceptivas; desequilíbrio vestibular e, até mesmo, déficits em nível cortical ${ }^{7-9}$.

As incidências das escolioses variam, dependendo da população estudada, do método de identificação e do grau da curva requerida. Por não existir uma padronização dos métodos de avaliação e dos registros dos resultados, são descritos dados que vão de $1-13 \%$, embora a incidência de escoliose com mais de $10^{\circ}$ seja de, aproximadamente, $2 \% \%^{7,10,11}$. A radiografia é o exame mais utilizado para diagnosticar as deformidades da coluna ou tentar avaliar o padrão de normalidade de suas curvaturas $^{12}$, sendo o método de Cobb o padrão utilizado para quantificar as medidas angulares ${ }^{13}$ e os métodos de Nash\&Moe ${ }^{14}$ e Raimondi ${ }^{15}$ para medida de rotação vertebral ${ }^{16}$. Devido aos efeitos radioativos cumulativos, a utilização desse exame deve ser limitada, uma vez que causam impactos prejudiciais à saúde ${ }^{17-19}$. Uma opção ao exame radiográfico é a avaliação por métodos não-invasivos que não expõe o indivíduo a riscos. Nesse contexto, atualmente, observa-se a utilização de diversas técnicas para essa finalidade, com a utilização de fios de prumo, réguas e escoliômetros ${ }^{8,20-23}$.

O teste de Adams tornou-se um procedimento padrão para detectar escoliose. Esse consiste na mensuração da gibosidade através da flexão anterior do tronco ${ }^{7,19}$. Estudos clássicos ${ }^{22,21}$ já utilizavam esse teste e um equipamento de madeira adaptado com nível d'água e réguas para mensurar a altura da gibosidade, em rastreamento de escoliose em grandes populações escolares. Todavia, esse método não levava em consideração a tridimensionalidade da curva. Justamente, devido a esse aspecto, nos estudos atuais, têm sido considerados os três planos de referência na avaliação da escoliose. Assim, o alinhamento no plano sagital tornou-se o foco de pesquisadores em busca de aumentar o entendimento sobre o assunto ${ }^{24-25}$. Algumas publicações relacionam o aspecto tridimensional, porém utilizando exames de radiografia ${ }^{4,16}$. Mesmo assim, ainda permanecem obscuras as reais relações entre tal alinhamento e deformidade da coluna.

Tendo em vista a importância da utilização de métodos não-invasivos e do comportamento da coluna vertebral em relação ao alinhamento no plano sagital, o presente estudo teve por objetivos avaliar tal alinhamento em indivíduos com alterações na medida da gibosidade, comparando com um grupo sem alterações significantes, através de um método simples, que não apresenta risco aos indivíduos e que pode ser facilmente utilizado na clínica. Além disso, visou testar a confiabilidade da utilização do instrumento não-invasivo e; verificar se existe uma correlação entre as medidas da gibosidade e os valores das curvaturas vertebrais.

\section{PROCEDIMENTOS METODOLÓGICOS}

Foram convidados a participar do estudo universitários com idade entre 18-25 anos. Os critérios de exclusão foram: escolioses adaptativas ou congênitas; cirurgia na coluna vertebral; gestantes e usuários de próteses ou órteses, obtidos através do relato do indivíduo e/ou documentação médica. Assim, foram incluídos 40 indivíduos, 19 mulheres e 21 homens. $\mathrm{O}$ estudo foi aprovado pelo Comitê de Ética em Pesquisa da Faculdade de Ciências e Tecnologia - Universidade Estadual Paulista, sob 
parecer $n^{\circ}$ 189/2007. Todos leram e assinaram um Termo de Consentimento Livre e Esclarecido, concordando participar do estudo.

Os participantes foram submetidos à mensuração da gibosidade e posterior medida das curvaturas no plano sagital (cifoses e lordoses). Essas avaliações foram realizadas, respectivamente, com auxílio de dois equipamentos de madeira adaptados com régua e nível d'água. $O$ primeiro (Figura 1A), fabricado pelas pesquisadoras, com bases na descrição de Surós ${ }^{26}$ para avaliação da gibosidade torácica, é constituído por dois níveis d'água encaixados numa madeira de dimensões: 30,5 x 5,0 x 2,0 cm (comprimento $\mathrm{x}$ largura $\mathrm{x}$ espessura). Apresenta um orifício de $6,0 \mathrm{~cm}$, que permite o encaixe e deslizamento perpendicular - para mensuração da altura da gibosidade, e paralelo - para buscar o ponto de maior rotação vertebral, de uma régua de madeira $(30 \mathrm{~cm})$. Já o segundo (Figura 1B), uma adaptação ao primeiro, desenvolvida pelas próprias pesquisadoras, para mensuração das curvaturas da coluna no plano sagital, é constituído por dois níveis d'água encaixados em uma madeira, de dimensões 35 × 5,0 x 2,0 cm (comprimento x largura $x$ espessura). Apresenta um orifício de $3,0 \mathrm{~cm}$, que permite o encaixe perpendicular de uma régua $(30 \mathrm{~cm}) . \mathrm{O}$ orifício não permite deslizamento em paralelo, somente perpendicular - para mensuração da profundidade da curva, sendo essa a principal diferença entre os dois instrumentos.

$\mathrm{Na}$ avaliação da gibosidade, o equipamento (Figura 1A) foi utilizado através do teste de Adams ${ }^{7,23}$. Para isso, foi solicitado ao sujeito que posicionasse seus pés sobre uma impressão plantar padronizada para todos os indivíduos (separada por uma distância de $10 \mathrm{~cm}$ e com uma abdução podal de $\left.16^{\circ}\right)^{27}$. Esse procedimento visou à manutenção do padrão postural fisiológico, evitando variações na medida. Em seguida, foi solicitado ao participante que realizasse a flexão anterior do tronco, deixando os membros superiores pendentes e relaxados. Tal instrumento foi colocado sobre a superfície do tronco, no ponto mais elevado da gibosidade e, mantendose o nível d’água equilibrado, foi verificada a altura da gibosidade em relação ao lado oposto.

Na presença de gibosidade significante, superior a 0,5 cm na coluna lombar e/ou $0,7 \mathrm{~cm}$ na coluna torácica - acusando provável escoliose, o indivíduo foi encaminhado ao grupo experimental (GE) ${ }^{22}$. Nos casos contrários, o indivíduo foi alocado no grupo controle (GC) - que conteve, portanto, sujeitos sem alterações significantes da gibosidade. Ao final dessas avaliações, o GE abrangeu 20 indi- víduos (nove mulheres e 11 homens - com idade média de 21,4 42,34 anos), e o GC, 20 indivíduos (10 mulheres e 10 homens - com idade média de 21,75 $\pm 2,57$ anos).

$\mathrm{Na}$ obtenção das medidas das curvaturas no plano sagital, foi solicitado ao sujeito posicionar seus pés da mesma forma que outrora e foram coletadas quatro medidas: cervical(C); torácica(D); lombar(E) e sacral(F) (Figuras 1C; 1D; 1E e 1F, respectivamente), com a utilização do instrumento ilustrado na figura $1 \mathrm{~B}$. $\mathrm{O}$ instrumento $\mathrm{B}$ foi posicionado no plano sagital medialmente ao osso occipital (C); à cifose torácica (D e E) e ao sacro (F). Em (C) e (E) o nível d'água encontrava-se voltado para cima, mantendo-se equilibrado. Já em (D) e (F), o nível d'água encontrava-se voltado para baixo e, também, equilibrado. Em (C) e (E) a régua foi deslizada até o ponto mais côncavo da lordose cervical, registrando-se a distância entre ela e o osso occipital (C) e, entre ela e a cifose torácica (E). Em (D) e (F) a régua foi deslizada até o ponto mais côncavo da lordose lombar, registrando-se a distância entre ela e a cifose torácica (D), e, entre ela e o sacro $(F)$.
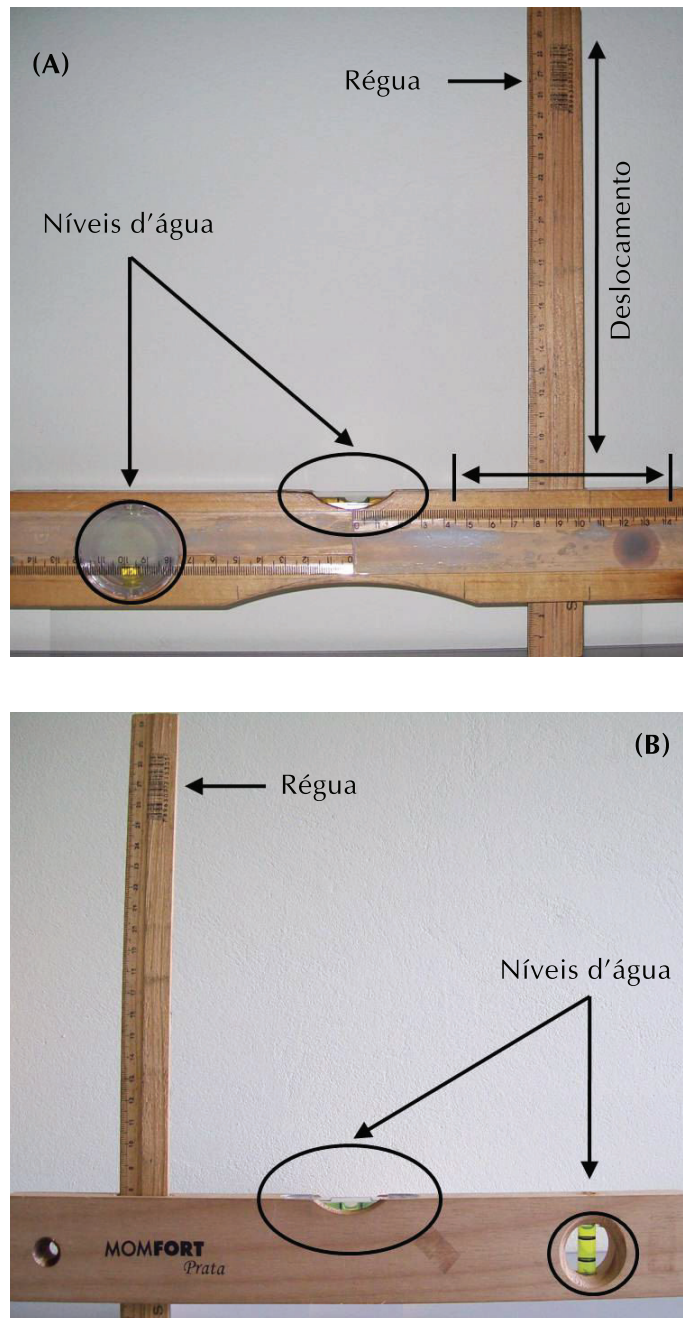


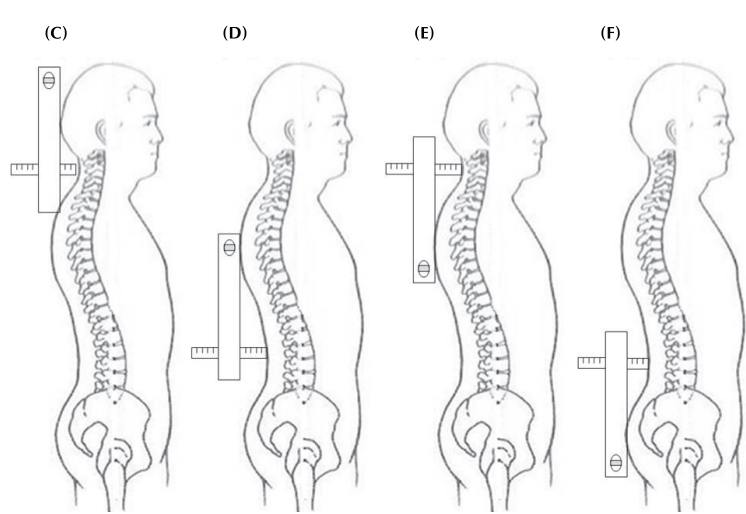

Figura 1. Equipamento e coleta das medidas das curvaturas no plano sagital.

(A): Equipamento utilizado para mensuração da gibosidade durante o teste de Adams; (B): equipamento utilizado para mensuração das curvaturas no plano sagital. Medidas das curvaturas: cervical(C); torácica(D); lombar(E) e sacral(F).

Essas medidas foram coletadas em duas ocasiões (coletas A e B), num intervalo de três a quatro dias, com intuito de confirmar a confiabilidade da técnica. Foi realizada sempre pelo mesmo avaliador, no mesmo local e período do dia, visando minimizar a variação intraobservador.

Inicialmente, foi realizada uma análise descritiva dos resultados para as medidas do plano sagital, com objetivo de verificar a distribuição das variáveis estudadas. Devido à característica de distribuição dos dados, foi utilizado o teste não-paramétrico de Mann-Whitney $(p<0,05)$ na realização das comparações entre os grupos, medidas e coletas. Já, para investigação de possíveis associações entre as variáveis estudadas (medidas da gibosidade $\mathrm{x}$ medidas das curvaturas) foi aplicado o teste de correlação de Spearman $(\mathrm{p}<0,05)$.

\section{RESULTADOS}

As características da análise descritiva dos resultados estão dispostas na figura 2 (I-A e II-A - GC e GE para a coleta A; I-B e II-B - GC e GE para a coleta $B$ ).

A tabela 1(A) apresenta as médias (desviospadrão) de cada variável e o resultado da análise comparativa. Com o teste Mann-Whitney, compararam-se os diferentes períodos de coleta (coletas A e B) e, os diferentes grupos de sujeitos (GE e GC). Notou-se diferença significante apenas nos valores de $C$ da coleta $A$, confirmada pela coleta $B$ somente para os dados da curvatura cervical. Já na tabela 1(B), pode ser observado que não existiu diferença nas coletas A e B para nenhuma medida do plano sagital, em nenhum dos grupos, demonstrando a confiabilidade dos dados.
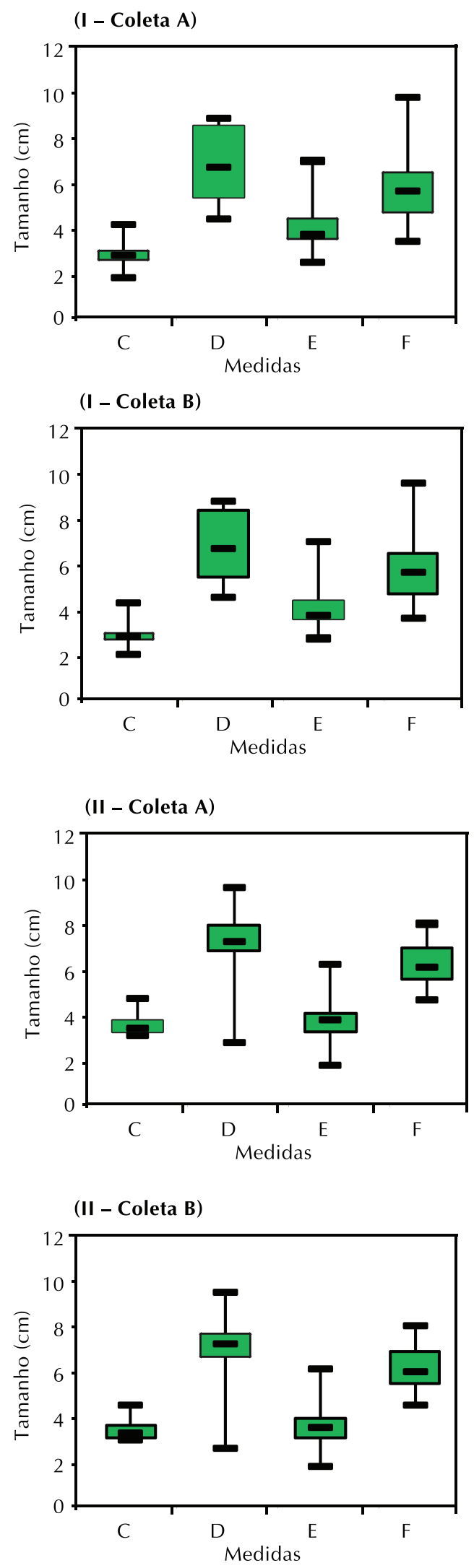

Figura 2. Valores da análise descritiva (amplitudes; medianas e percentis a $25 \%$ e $75 \%)$. (I): Grupo controle $(n=20)$ e (II): grupo experimental $(n=20)$, para as coletas (a): primeiro dia e (b): segundo dia. (C): medida cervical; (D) medida torácica; (E): medida lombar e (F): medida sacral. 
Os resultados do teste de correlação linear podem ser observados na tabela 2, que mostra que os valores da medida $\mathrm{D}$ e gibosidade torácica no $\mathrm{GC}$, e da medida F e gibosidade torácica no GE apresentaram correlação linear significante. Essa correlação admitiu coeficiente positivo, gerando uma reta crescente (Figuras 3A e 3B). No caso da medida E e gibosidade torácica no GE, foi encontrada correlação linear também significante, porém de valor negativo, gerando uma reta decrescente (Figura 3C).

Tabela 1 - (A): Comparações entre as médias (desvios-padrão) das medidas das curvaturas vertebrais entre os GE e GC, no primeiro e no segundo dia de coleta. (B): Comparações entre as médias (desvios-padrão) das medidas das curvaturas vertebrais, no primeiro e no segundo dia de coleta, para os GE e GC - análise da confiabilidade intraexaminador das medidas das curvaturas vertebrais.

(A)

\begin{tabular}{|c|c|c|c|c|}
\hline \multirow[b]{2}{*}{$\frac{\frac{\pi}{0}}{0}$} & \multirow[b]{2}{*}{$\begin{array}{l}\frac{n}{0} \\
\frac{.0}{0} \\
\frac{d}{2}\end{array}$} & \multicolumn{3}{|c|}{ Grupos } \\
\hline & & 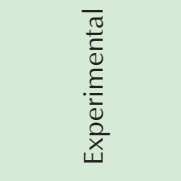 & $\begin{array}{l}\frac{0}{0} \\
\frac{0}{0} \\
0\end{array}$ & $\begin{array}{l}\frac{1}{0} \\
\frac{\pi}{20} \\
\frac{1}{2}\end{array}$ \\
\hline \multirow{4}{*}{ a } & $\mathrm{C}$ & $3,465(0,558)$ & $2,895(0,610)$ & $0,0005^{*}$ \\
\hline & $\mathrm{D}$ & $7,015(1,563)$ & $6,775(1,650)$ & 0,6260 \\
\hline & E & $3,480(1,087)$ & $4,150(1,286)$ & 0,2843 \\
\hline & $\mathrm{F}$ & $6,050(1,034)$ & $5,795(1,443)$ & 0,3034 \\
\hline \multirow{4}{*}{ b } & C & $3,475(0,545)$ & $2,890(0,578)$ & $0,0002^{*}$ \\
\hline & D & $7,005(1,555)$ & $6,765(1,599)$ & 0,5789 \\
\hline & $\mathrm{E}$ & $3,540(1,021)$ & $4,160(1,217)$ & 0,2280 \\
\hline & $\mathrm{F}$ & $6,080(1,027)$ & $5,785(1,400)$ & 0,2498 \\
\hline
\end{tabular}

(a): Primeiro dia de coleta; (b): segundo dia de coleta; $C$ : Medida cervical; D: Medida torácica; E: Medida lombar; F: Medida sacral. ${ }^{*} \mathrm{p}<0,05$

(B)

\begin{tabular}{|c|c|c|c|c|}
\hline \multirow{2}{*}{$\begin{array}{l}\frac{n}{0} \\
\frac{\pi}{0} \\
\frac{0}{2} \\
\sum \\
\Sigma\end{array}$} & \multirow[b]{2}{*}{$\begin{array}{l}\stackrel{n}{0} \\
\stackrel{2}{2} \\
\frac{2}{U}\end{array}$} & \multicolumn{3}{|c|}{ Coletas } \\
\hline & & a & $b$ & $\begin{array}{l}\frac{1}{0} \\
\frac{0}{\pi} \\
1 \\
2\end{array}$ \\
\hline \multirow{2}{*}{$\mathrm{C}$} & $\mathrm{CO}$ & $2,895(0,610)$ & $2,890(0,578)$ & 1,0000 \\
\hline & EXP & $3,465(0,558)$ & $3,475(0,545)$ & 0,9460 \\
\hline \multirow{2}{*}{$\mathrm{D}$} & $\mathrm{CO}$ & $6,775(1,650)$ & $6,765(1,599)$ & 0,8812 \\
\hline & EXP & $7,015(1,563)$ & $7,005(1,555)$ & 0,9784 \\
\hline \multirow{2}{*}{$\mathrm{E}$} & $\mathrm{CO}$ & $4,150(1,286)$ & $4,160(1,217)$ & 0,8378 \\
\hline & EXP & $3,480(1,087)$ & $3,540(1,021)$ & 0,9892 \\
\hline \multirow{2}{*}{$\mathrm{F}$} & $\mathrm{CO}$ & $5,795(1,443)$ & $5,785(1,400)$ & 0,9459 \\
\hline & EXP & $6,050(1,034)$ & $6,080(1,027)$ & 0,7652 \\
\hline
\end{tabular}

EXP: grupo experimental; CO: grupo controle; (a): Primeiro dia de coleta; (b): segundo dia de coleta; C: Medida cervical; D: Medida torácica; E: Medida lombar; F: Medida sacral.
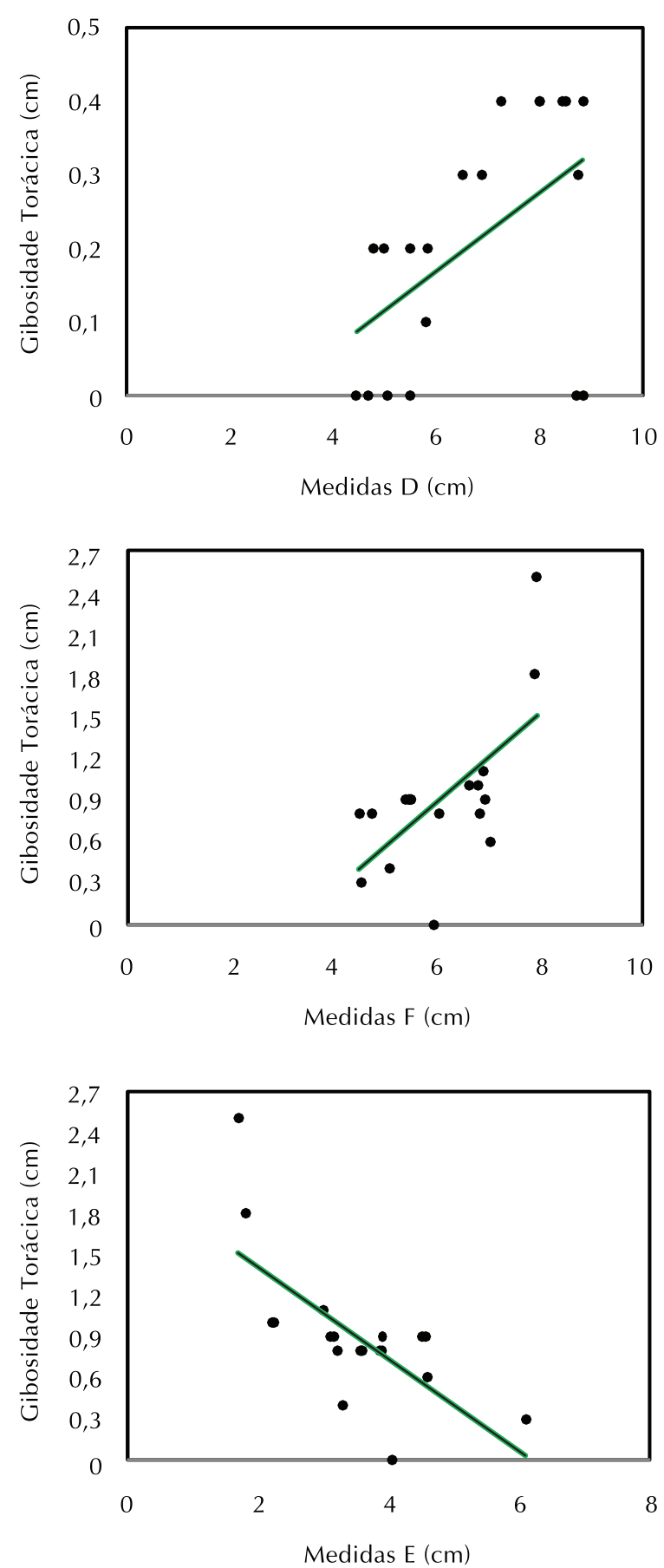

Figura 3. Correlações lineares encontradas entre as curvaturas sagitais e a gibosidade.

(A): Correlação entre gibosidade torácica e a medida D (torácica) no grupo controle $(r=0,495)$; (B): Correlação entre gibosidade torácica e a medida $\mathrm{F}$ (sacral) no grupo experimental $(r=0,534) ;(C)$ : Correlação entre gibosidade torácica e a medida E (lombar) no grupo experimental $(r=-0,683)$.

\section{DISCUSSÃO}

A medida da gibosidade foi realizada segundo orientações de Ferreira et al. ${ }^{7}$; Salate et al. ${ }^{8}$ e Ferreira e Defino ${ }^{23}$ que utilizaram o mesmo instrumento nessa avaliação. Do total de indivíduos avaliados, $87,5 \%$ eram portadores de algum tipo de gibosidade, com 
Tabela 2. Valores do teste de correlação de Spearman ( $\rho$ e pvalor) entre as medidas das curvaturas no plano sagital e das gibosidades nos grupos controle (A) e experimental (B).

\begin{tabular}{|c|c|c|c|c|c|c|}
\hline \multicolumn{7}{|c|}{ GRUPO CONTROLE } \\
\hline $\begin{array}{l}\frac{0}{0} \\
\frac{\pi}{0} \\
\frac{\pi}{0} \\
\frac{0}{0} \frac{0}{n} \\
\sum \frac{0}{0}\end{array}$ & 0 & $山$ & $\sqcup$ & $\stackrel{0}{\stackrel{0}{u}}$ & $\underset{⿱ ㇒}{\vec{b}}$ & $\frac{0}{\dot{1}}$ \\
\hline \multicolumn{7}{|l|}{$\mathrm{C}$} \\
\hline$\rho$ & 0,15 & 0,20 & $-0,07$ & 0,19 & $-0,07$ & 0,33 \\
\hline$p$ & 0,50 & 0,39 & 0,74 & 0,40 & 0,73 & 0,14 \\
\hline \multicolumn{7}{|l|}{$\mathrm{D}$} \\
\hline$\rho$ & & 0,50 & $-0,37$ & 0,49 & $-0,24$ & 0,02 \\
\hline $\mathrm{p}$ & & $0,02^{*}$ & 0,09 & $0,02 *$ & 0,30 & 0,93 \\
\hline \multicolumn{7}{|l|}{$\mathrm{E}$} \\
\hline$\rho$ & & & $-0,37$ & $-0,02$ & 0,26 & 0,18 \\
\hline$p$ & & & 0,10 & 0,91 & 0,26 & 0,44 \\
\hline \multicolumn{7}{|l|}{$\mathrm{F}$} \\
\hline$\rho$ & & & & 0,12 & 0,05 & $-0,33$ \\
\hline $\mathrm{p}$ & & & & 0,59 & 0,79 & 0,14 \\
\hline \multicolumn{7}{|l|}{ G-TO } \\
\hline$\rho$ & & & & & $-0,28$ & 0,10 \\
\hline $\mathrm{p}$ & & & & & 0,21 & 0,66 \\
\hline \multicolumn{7}{|l|}{ G-TL } \\
\hline$\rho$ & & & & & & $-0,05$ \\
\hline$p$ & & & & & & 0,82 \\
\hline \multicolumn{7}{|c|}{ GRUPO EXPERIMENTAL } \\
\hline $\begin{array}{l}\frac{0}{0} \\
\frac{\pi}{0} \\
\frac{0}{0} \\
\frac{0}{0} \\
\sum \frac{0}{0} \\
\sum \frac{0}{0}\end{array}$ & 0 & $山$ & $\sqcup$ & $\stackrel{0}{\frac{0}{0}}$ & $\underset{\dot{U}}{\vec{F}}$ & $\frac{0}{u}$ \\
\hline \multicolumn{7}{|l|}{ C } \\
\hline$\rho$ & 0,004 & 0,20 & 0,13 & $-0,36$ & 0,38 & 0,13 \\
\hline $\mathrm{p}$ & 0,98 & 0,39 & 0,55 & 0,11 & 0,09 & 0,56 \\
\hline \multicolumn{7}{|l|}{$\mathrm{D}$} \\
\hline$\rho$ & & 0,63 & $-0,45$ & $-0,34$ & $-0,35$ & $-0,19$ \\
\hline$p$ & & $0,002 *$ & $0,04 *$ & 0,13 & 0,12 & 0,40 \\
\hline \multicolumn{7}{|l|}{$E$} \\
\hline$\rho$ & & & $-0,49$ & $-0,68$ & 0,23 & $-0,30$ \\
\hline$p$ & & & $0,02 *$ & $0,00^{*}$ & 0,32 & 0,19 \\
\hline \multicolumn{7}{|l|}{$\mathrm{F}$} \\
\hline$\rho$ & & & & 0,53 & 0,24 & 0,16 \\
\hline$p$ & & & & $0,01 *$ & 0,29 & 0,49 \\
\hline \multicolumn{7}{|l|}{ G-TO } \\
\hline$\rho$ & & & & & $-0,29$ & 0,088 \\
\hline $\mathrm{p}$ & & & & & 0,196 & 0,705 \\
\hline \multicolumn{7}{|l|}{ G-TL } \\
\hline$\rho$ & & & & & & $-0,13$ \\
\hline$p$ & & & & & & 0,55 \\
\hline
\end{tabular}

GC: Grupo controle; GE: Grupo experimental; $\rho$ : coeficiente de Spearmann; C: Medida cervical; D: Medida torácica; E: Medida lombar; F: Medida sacral; G-TO: Gibosidade Torácica; G-TL: Gibosidade Tóraco-lombar; G-LO: Gibosidade Lombar. ${ }^{*}<<0,05$.

predominância no hemitórax direito (85,71\%). Dentre as gibosidades, $42,85 \%$ eram de $0,40 \mathrm{~cm}$ ou menos, constituindo, assim, gibosidades de caráter fisiológico ${ }^{21-22}$. Ainda, no presente estudo, houve diferença significante entre GC e GE apenas para a medida C, ou seja, em níveis cervicais (tabela 1 A). Isso significa que nas medidas $\mathrm{D}, \mathrm{E}$ e $\mathrm{F}$ não foram encontradas diferenças relevantes entre os grupos. Considerando que o presente estudo não analisou medidas radiográficas, não foi objetivo realizar uma análise mais aprofundada e, por conseguinte, mais invasiva dessas variáveis.

Muitos estudos descrevem a intercalação de medidas não invasivas às radiográficas, a fim de se obter um bom parâmetro quantitativo de acompanhamento, sem expor os pacientes aos riscos decorrentes da radiação excessiva ${ }^{15-16}$. Leroux et al. ${ }^{28}$ utilizaram métodos não invasivos para mensurar cifose e lordose em 124 mulheres, através de marcadores na pele e videografia, correlacionando-os com medidas radiológicas. Relataram que, mesmo sem obter sempre todas as medidas antropométricas, encontraram um bom coeficiente intraclasse - confiabilidade, de forma que concluíram ser confiável o meio de avaliação pesquisado. No presente estudo, a variação entre medidas do plano sagital e coletas não foi significante, nem no GC nem no GE (Tabela 1B). A insignificância da variação intraobservador foi um forte indicativo de validade concorrente entre os valores obtidos para as coletas feitas por um único avaliador.

Estudos que também relacionam medidas não-invasivas às radiológicas descrevem algumas associações que devem ser levadas em consideração. Ferreira e Defino ${ }^{23}$ observaram correlações mais satisfatórias ao se confrontar a gibosidade torácica com o ângulo de Cobb e, também, ao se comparar rotação vertebral pelos métodos Nash\&Moe e Raimondi. As associações da medida da gibosidade com ângulo de Cobb também foram satisfatórias na região torácica e tóraco-lombar direita, na pesquisa de Ferreira et al. ${ }^{7}$,e, nesse mesmo estudo, as medidas de gibosidade com rotação vertebral se correlacionaram melhor na região lombar. Já na pesquisa de Salate et $a .^{8}$ observou-se melhor correlação das medidas de gibosidade com o ângulo de Cobb, na região tóraco-lombar e com a rotação vertebral nas regiões tóraco-lombar e lombar. Sendo assim, tais estudos, em âmbito geral, corroboram entre si, todavia, levando-se em consideração aspectos mais específicos, mesmo com o uso de radiografias, ainda remanescem conclusões contraditórias.

No presente estudo, as medidas de associação foram realizadas, visando correlacionar a mensuração da gibosidade aos valores das curvaturas no plano 
sagital. Podem ser destacados, em ambos os grupos, correspondência linear significante positiva entre as medidas D e E (Tabela 2). Considerando as posições em que são obtidas tais variáveis (ambas com base na cifose torácica), o resultado mostra coerência entre as medidas. Exclusivamente no GE, ocorreu relação linear significante para as medidas $\mathrm{E}$ (negativa) e $\mathrm{F}$ (positiva) em relação à gibosidade torácica (Figuras 3B e 3C). Nesse grupo houve, ainda, correspondência linear significante negativa entre a medida $\mathrm{Fe}$ as medidas De E (Tabela 2B). Nessa última relação, pode ter ocorrido um desequilíbrio no alinhamento vertebral influenciado por uma possível curvatura escoliótica, uma vez que a associação só foi significante no GE.

Öhlén et al. ${ }^{25}$ realizaram um estudo com 127 sujeitos com escoliose e 92 controles. Em pacientes com curvas torácicas, os graus de cifose torácica e de lordose lombar foram significantemente menores que os encontrados em controles. Esses achados discordam dos resultados do presente estudo, no qual não houve diferença significativa em níveis torácicos e lombares entre os GE e GC. Contudo, os autores afirmaram não haver tendência para diminuir a cifose ou a lordose quando a deformidade escoliótica aumenta, mas concluíram que a presença de dorso plano acarreta o surgimento da escoliose.

Mac-Thiong et al..$^{29-30}$ realizaram estudos sobre alinhamento sagital da coluna, relação com movimentos pélvicos e análise desses parâmetros em adolescentes com escoliose idiopática. Os resultados obtidos mostraram que a medida da incidência pélvica tende a aumentar entre os quatro e 18 anos de idade e que a inclinação pélvica e a lordose lombar também aumentam com a idade até que se alcance um equilíbrio sagital adequado. Entretanto, esses autores não encontraram diferença significante entre grupos com escoliose e controle para estes parâmetros. A cifose torácica mostrou dependência com a deformidade da coluna, de forma que era relativamente menor quando havia curva escoliótica torácica. Sendo assim, esses resultados ${ }^{29-30}$ concordam com o presente estudo, no sentido de constatar que curvas escolióticas torácicas são um parâmetro importante de avaliação, já que mostraram ter boa correlação com os outros aspectos avaliados.

\section{CONCLUSÃO}

Pode-se concluir que os grupos, divididos em controle e experimental em função da medida da gibosidade, apresentaram diferença apenas para a curvatura cervical e a técnica utilizada mostrou-se confiável. Algumas medidas da gibosidade correlacionaram-se com as medidas no plano sagital. Embora, os resultados tenham apontado confiabilidade, inferindo possível escoliose, não se deve diagnosticá-la na ausência de exames radiográficos. Por esse motivo, o fato do grupo experimental ter sido composto por indivíduos que apresentavam escoliose, porém sem a confirmação radiográfica, implicou uma limitação do estudo. As medidas não-invasivas devem ser uma alternativa ou complementação nas avaliações posturais, pois possibilitam o acompanhamento da evolução das deformidades, através de medida simples, rápida e sem prejuízo da salubridade do avaliado na clínica. Ainda se fazem necessárias pesquisas futuras, com mais de um avaliador e que as correlacione com as medidas radiográficas da cifose torácica e da lordose lombar, para consolidação da eficácia científica do método.

\section{REFERÊNCIAS BIBLIOGRÁFICAS}

1. Sacco ICN,Tanaka C. Cinesiologia e biomecânica dos complexos articulares. Rio de Janeiro: Guanabara Koogan; 2008.

2. Magee DJ. Disfunção musculoesquelética. São Paulo: Manole; 2005.

3. Neumann DA. Cinesiologia do sistema musculoesquelético: fundamentos para reabilitação. Rio de Janeiro: Guanabara Koogan; 2006.

4. Perdriolle R, Le Borgne P, Dansereau J, Guise J, Labelle H. Idiopathic scoliosis in three dimensions: a succession of two-dimensional deformities. Spine 2001;26(15):2719-26.

5. Castelein RM, Van Dieën JH, Smith TH. The role of dorsal shear forces in the pathogenesis of adolescent idiopathic scoliosis-a hypothesis. Med Hypotheses 2005;65:501-8.

6. Hebert S, Xavier R. Ortopedia e traumatologia: princípios e prática. São Paulo: Artmed; 2003.

7. Ferreira DMA, Suguikawa TR, Pachioni CAS, Fregonesi CEPT, Camargo MR. Rastreamento escolar da escoliose:medida para o diagnóstico precoce. Rev Bras Crescimento Desenvolvimento Hum 2009;19:357-68.

8. Salate ACB, Aroni FC, Ferreira DMA. Estudo da evolução à curto prazo da escoliose por meio de mensurações da gibosidade, radiográficas e da dor em adolescentes e adultos jovens. Rev Bras Fisioter 2003;7:39-44.

9. Simoneau M, Richer N, Mercier P, Allard P, Teasdale N. Sensory deprivation and balance control in idiopathic scoliosis adolescent. Exp Brain Res 2006;170(4):576-82.

10. Liu S-L, Huang D-S. Scoliosis in China. Clin Orthop Relat Res 1996;323 (1):113-8.

11. Coillard C, Rivard CH. Vertebral deformities and scoliosis. Eur Spine J 1996;5:91-100.

12. Ramos E, Reis DC, Esteves AC. Análise cinemática da marcha em portadores de escoliose idiopática. Rev Bras Cineantropom Desempenho Hum 2006;8:85-92.

13. Morrissy RT, Goldsmith ES, Hall EC, Cowie GH. 
Meansurament of the Cobb angle on radiographs of patients who have scoliosis. Evaluation of intrinsic error J Bone Joint Surg 1990;72(3):320-7.

14. Nash CL, Moe JH. A study of vertebral rotation. J Bone Joint Surg. 1969;51-A(2):223-9.

15. Weiss HR. Measurement of vertebral rotation: Pedriolle versus Raimondi Eur Spine J 1995;4:34-8.

16. Defino HLA, Araújo PHM. Estudo comparativo da medida da rotação vertebral pelos métodos de Nash\&Moe e método de Raimondi. Acta Ortop Bras 2004;12:167-73.

17. Kogutt MS, Warren FH, Kalmar JA. Low dose imaging of scoliosis: use of a computed radiographic imaging system. Pediatr Radiol 1989;20(1-2):85-6.

18. Nash CL, Gregg EC, Brown RH, Pillai K. Risks of exposure to $\mathrm{x}$-rays in patients undergoing long-term treatment for scoliosis. J Bone Joint Surg 1979;61A(3):371-4.

19. Teixeira FA, Carvalho GA. Confiabilidade e validade das medidas da cifose torácica através do método flexicurva. Rev Bras Fisioter 2007;11:199-204.

20. Côté P, Kreitz BG, Cassidy JD, Dzus AK, Martel J. A study of the diagnostic accuracy and reliability of scoliometer and Adams forward bend test. Spine 1998;23:796-803.

21. Soucacos PN, Soucacos PK, Zacharis K, Beris AE, Xenakis TA. School-screening for scoliosis:a prospective epidemiological study in northwestern and central Greece. J Bone Joint Surg 1997;79-A:1498-503.

22. Vercauteren M, Van Beneden M, Verplaetse R, Croene P, Uyttendaele D, Verdonk R. Trunk asymmetries in a Belgian school population. Spine 1982;7(8):55-62.

23. Ferreira DMA, Defino HLA. Avaliação quantitativa da escoliose idiopática: concordância das mensurações de gibosidade e correlações com medidas radiológicas. Rev Bras Fisioter 2001;5(2):73-86.
24. Bethune DD, Broekhoven LH, Kung E, Snewing DG. Statistical method for evaluating human thoracolumbar spinal curves in the sagittal plane:a preliminary report. Arch Phys Med Rehabil 1986;67:590-4.

25. Öhlén G, Aaro S, Bylund P. The sagittal configuration and mobility of the spine in idiopathic scoliosis. Spine 1988;13:413-6.

26. Surós J. Semiologia medica y tecnica exploratoria. Barcelona: Salvat; 1977.

27. González LJ, Sreenivasan SV, Jensen JL. A procedure to determine equilibrium postural configurations for arbitrary locations of the feet. J Biomech Eng 1999;121:644-9.

28. Leroux MA, Zabjek K, Simard G, Badeaux J, Coillard $\mathrm{C}$, Rivard C. A noninvasive anthropometric technique for measuring kyphosis and lordosis-an application for idiopathic scoliosis. Spine 2000;25:1689-94.

29. Mac-Thiong J-M, Berthonnaud E, Dimar JR, Betz RR, Labelle H. Sagittal alignment of the spine and pelvis during growth. Spine 2004;29:1642-7.

30. Mac-Thiong J-M, Labelle H, Charlebois M, Huot M-P, Guise JA. Sagittal plane analysis of the spine and pelvis in adolescent idiopathic scoliosis according to the coronal curve type. Spine 2003;28:1404-9.

Endereço para correspondência

Dalva Minonroze Albuquerque

Departamento de Fisioterapia

R. Roberto Simonsen, 305

CEP 19060-900 - Presidente Prudente,SP. Brasil

E-mail: dalva@fct.unesp.br 